\title{
Relationship between Prenatal Depression with Social Support and Marital Satisfaction
}

\section{A R T I C L E I N F O}

\section{Article Type}

Original research

\section{Authors}

Noury R.* $P h D$,

Karimi N. ${ }^{1} B S C$

Mohammadi M. ${ }^{2} M D$

\section{How to cite this article}

Noury R, Karimi N, Mohammadi

M. Relationship between Prenatal Depression with Social Support and Marital Satisfaction. Sarem Journal of Reproductive Medicine. 2017;1(4):153-157.
*Psychology Department, Psychology \& Educational Sciences Faculty, Kharazmi University, Tehran, Iran ${ }^{1}$ Psychology \& Educational Sciences Faculty, Kharazmi University, Tehran, Iran

${ }^{2}$ Sarem Fertiliy and Infertility Research Center (SAFIR), Sarem Women's Hospital, Tehran, Iran

\section{Correspondence}

Address: -

Phone: -

Fax: -

rynoury@yahoo.com

\section{Article History}

Received: April 20, 2016

Accepted: October 16,2016

ePublished: November 15, 2017

\section{A B S T R A C T}

Aims Depression during pregnancy is one of the risk factors for postpartum depression and other complications of mothers and infants. The objective of this study was to determine the relationships between social support and marital satisfaction with depression during pregnancy.

Materials \& Methods This descriptive-correlational study was conducted on 70 pregnant women who referred to private clinics of Karaj (Iran) during a period of 6 months to receive prenatal care in the last 3 months of gestation. The information of the women was collected by Beck depression inventory (BDI-II), Enrich marital satisfaction and Wax social support (SS-A) questionnaires. The data were analyzed by correlation coefficient, regression variance analysis (univariate and multivariate) using SPSS software.

Findings There were significant correlations between depression during pregnancy with social support ( $r=-0.368)$ and marital satisfaction ( $r=-0.316)$. Social support and marital satisfaction predicted $15.3 \%$ of prenatal depression's variances. Social support was a better predictor for prenatal depression than marital satisfaction.

Conclusion There are significant correlations between depression during pregnancy with social support and marital satisfaction. Social support has a more important role in the prediction of depression during pregnancy than marital satisfaction.

\section{Keywords Depression Disorder; Social Support; Marital Satisfaction; Beck Depression; ENRICH Marital Satisfaction Questionnaire; Wax Social Support Questionnaire}

\section{I T A T I O N L I N K S}

[1] Frequancy and associated factors for anxiety and depression in pregnant women: A hospital ... [2] Cohort study of depressed mood during pregnancy and ... [3] Antenatal risk factors for postnatal depression ... [4] Sociodemographic predictors of antenatal and ... [5] The course of anxiety and depression through pregnancy ... [6] Depression and anxiety in Singaporean ... [7] Study of type of delivery with incidence of early ... [8] Rates and risk factors associated with depressive ... [9] Prevalence of depression during pregnancy ... [10] Perinatal depression: A systematic ... [11] Sucide: The leading cause of ... [12] Antenatal anxiety predicts child behavioral/emotional problems independently ... [13] Proactive nursing: The evolution of a task force to help women with ... [14] Depression and anxiety during pregnancy: A risk factor for obstetric, fetal and ... [15] Antenatal risk factors for postpartum depression: A synthesis ... [16] Antenatal maternal anxiety is linked with atypical handedness ... [17] Prevalence and factors associated with development of antenatal and postnatal ... [18] Antenatal depression strongly predicts postnatal depression ... [19] Risk factors for antenatal depression, postnatal depression and parenting ... [20] Prenatal prediction of poor maternal and offspring ... [21] The association of psychosocial factors and ... [22] Association between mental stress and ... [23] Health psychology: Biopsychosocial ... [24] The buffering effect of relationship satisfaction on emotional ... [25] The association between depression and marital ... [26] Antenatal risk factors for postnatal depression: A prospective ... [27] Antenatal depression in East Asia: A review of ... [28] Social support and antenatal depression in extended and nuclear family environments ... [29] Comparison of postpartum depression after natural delivery and emergency sezarian in first pregnant women who refer ... [30] Comparison of postpartum depression between occupatied ... [31] Causal relationship between stressful life events ... [32] Measuring social support in pregnancy ... [33] Evaluation of a social support measure that ... [34] Relationships between marital satisfaction ... [35] Study of scientific model of personality ... [36] Depression in pregnancy and ... [37] Intimate realationships and depression: Is there ... 


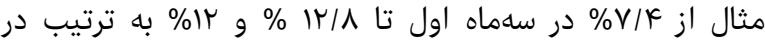

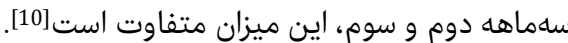

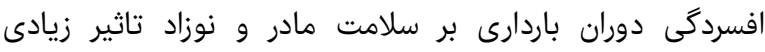

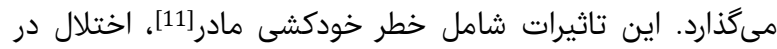

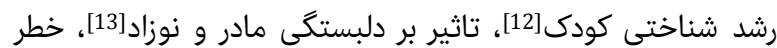

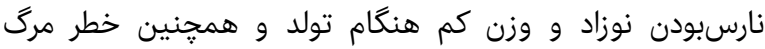

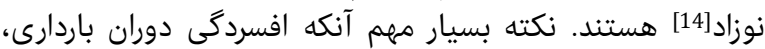
يكى از مهمترين متغيرهاى يِيشبين افسردگى يس إنى از زايمان

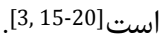

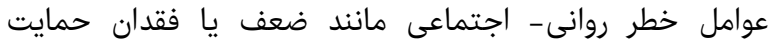

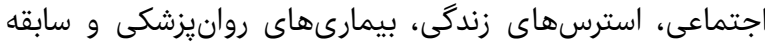

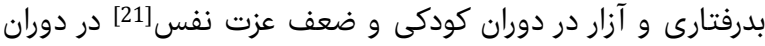

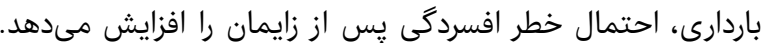

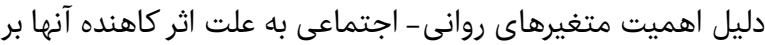

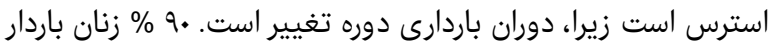

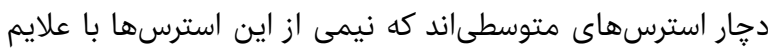

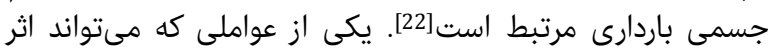

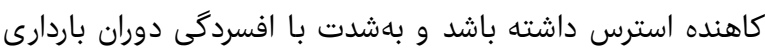

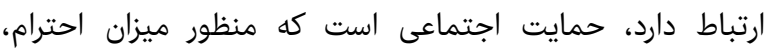

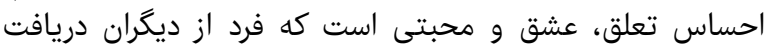

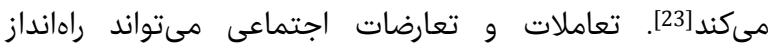

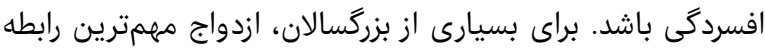

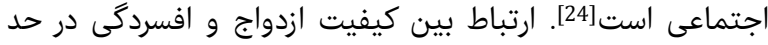

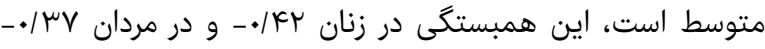

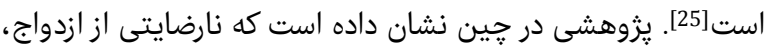

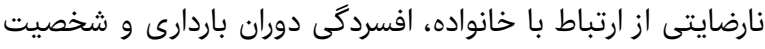

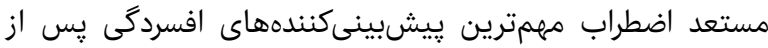

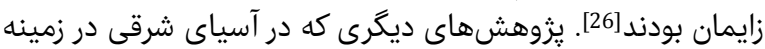

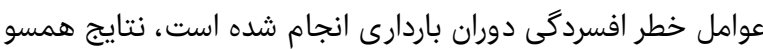

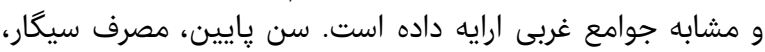

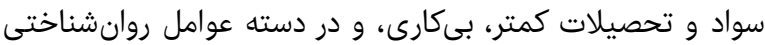

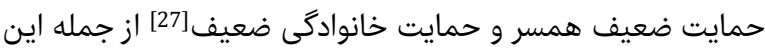

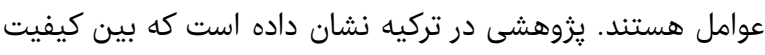

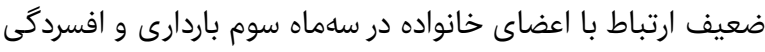

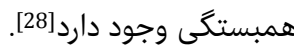

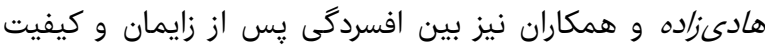

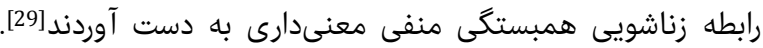

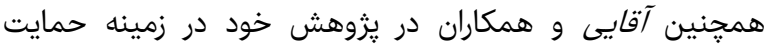

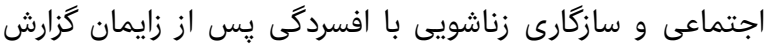

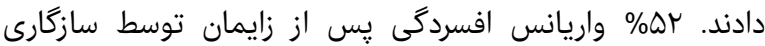

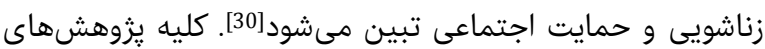

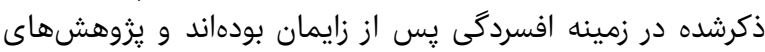

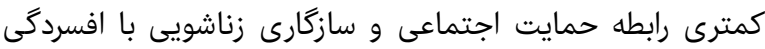

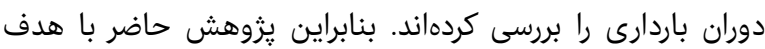

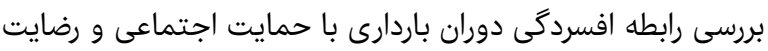

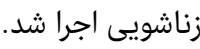

\section{مواد و روشها}

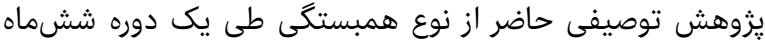

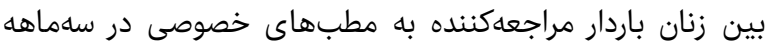

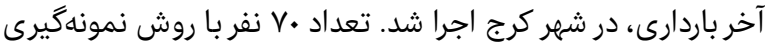

رابطه افسردگى دوران باردارى با حمايت اجتماعى روانى

و رضايت زناشويى افسيى دودري

PhD " ربابه نورىى

كروه روانشناسى، دانشكده روانشناسى و علوم تربيتى، دانشكاه خوارزمى، تهران، ايران

نجمه كريمى BSc ت نجران

دانشكده روان شناسى و علوم تربيتى، دانشكاه خوارزمى، تهران، ايران

MD مزگًان محمدى روان

مركز تحقيقات بارورى و نابارورى صارم، بيمارستان فوق تخصصى صارم، تهران،

جكيده

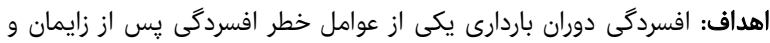

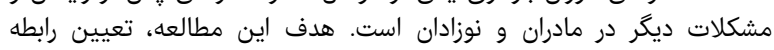

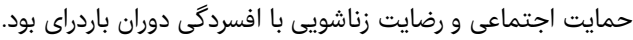

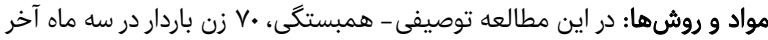

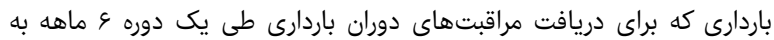

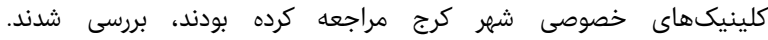

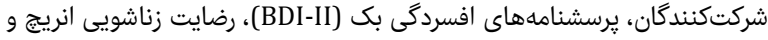

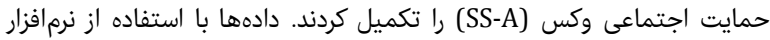
SPSS

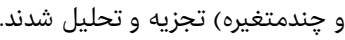

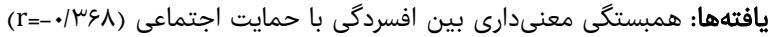

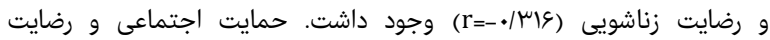

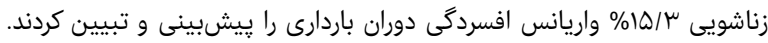

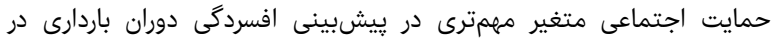

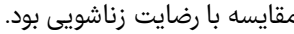

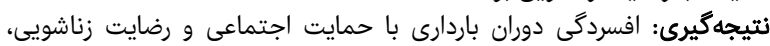

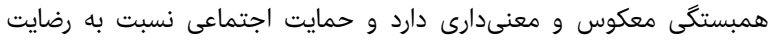

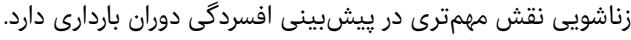

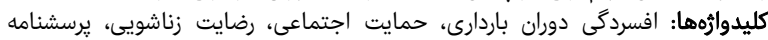

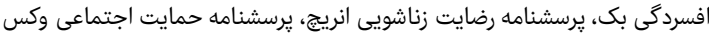

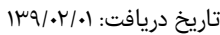

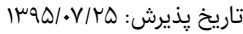

نويسنده مسئول: تأن

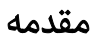

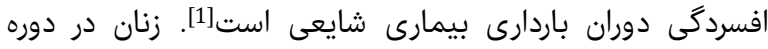

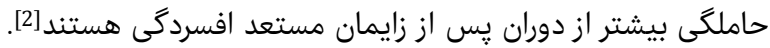

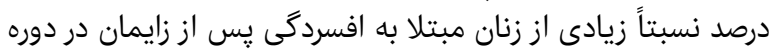

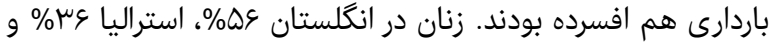

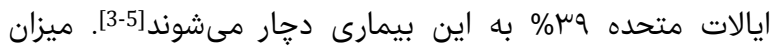

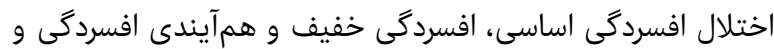

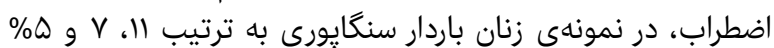

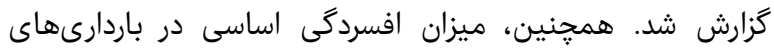

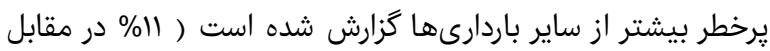

[6]

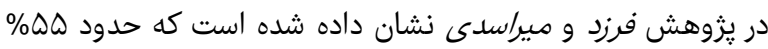

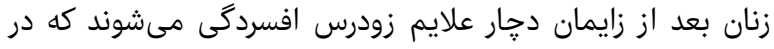

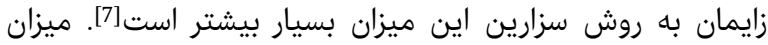

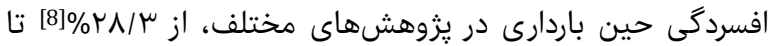

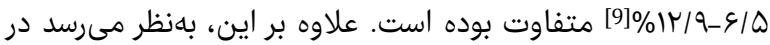

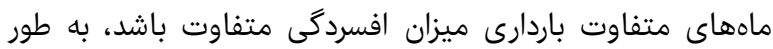


10Q رابطه افسردگى دوران باردارى با حمايت اجتماعى و رضايت زناشويى

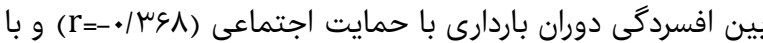

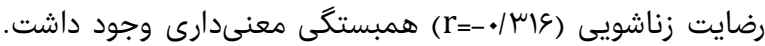

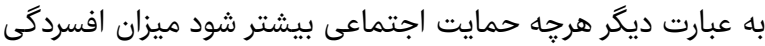

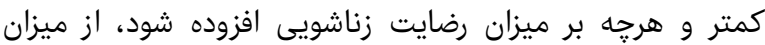
افسردگى دوران باردارى كاسته مى مئشان.

جدول ا) فراوانى مطلق و فراوانى نسبى (ويزگى هاى دموگرافيك شركتكنندگان

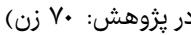

\begin{tabular}{|c|c|c|}
\hline فراوانى (درصد) & متغير & \\
\hline & & تحصيلات \\
\hline $1(1 / F)$ & ابتدايى & \\
\hline$\varepsilon(\Lambda / \varepsilon)^{-}$ & راهنمايى & \\
\hline$\mu_{q}(\Delta Q / \vee)$ & دييلم & \\
\hline$V(1 . / \cdot)$ & فوق دييلم & \\
\hline $18(Y K / Q)$ & ليسانس & \\
\hline $1(1 / k)$ & فوق ليسانس & \\
\hline & & شغل \\
\hline$Q \cdot(V Y / F)$ & خانهدار & \\
\hline$r \cdot(Y N / 9)$ & شاغل & \\
\hline & & ماه باردارى \\
\hline$\mu \mid(k F / \mu)$ & هفتم & \\
\hline $10(K I / F)$ & هشتم & \\
\hline$\mu F(\mu K / \mu)$ & نهم & \\
\hline
\end{tabular}

هاء از نمره افسردگى دوران باردارى از طريق حمايت اجتماعى و

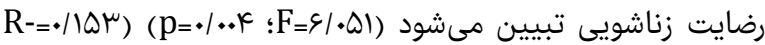

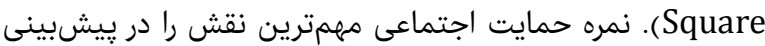

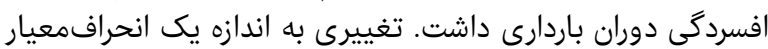

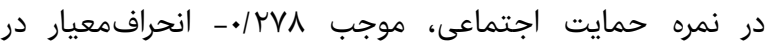

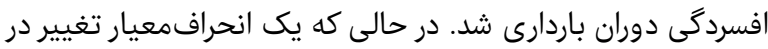

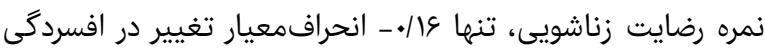

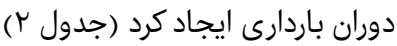

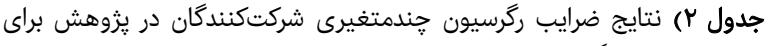

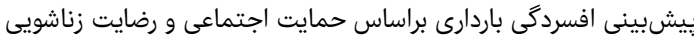

\begin{tabular}{|c|c|c|c|c|c|}
\hline معنىدارى & t ميزان t & ضرايب نمرات & خطانداى & ضرايب نمرات (B) & متغير \\
\hline.$/ . k k$ & $-r / . k q$ & $-\cdot /$ YYA &.$\| k F$ & -./rqs & اجتماعى \\
\hline$\cdot|\mu k|$ & $-1 / / \Lambda \mu$ &.$- / 19$. &.$/ 1 r$. & $-\cdot / l k r$ & رضايت زناشويى \\
\hline $\mid . . .1$ & F/VGF & - & $\Lambda / \Delta १ \Lambda$ & $k \cdot / 99 \Delta$ & عدد ثابت \\
\hline
\end{tabular}

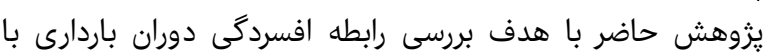

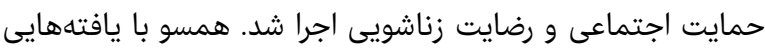

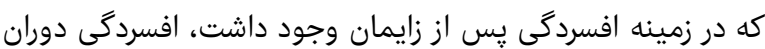

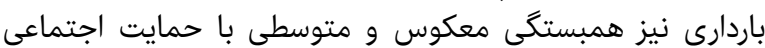

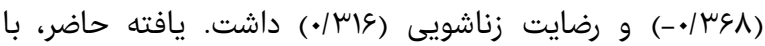

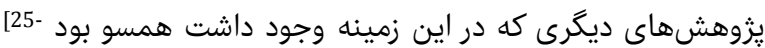

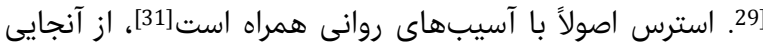

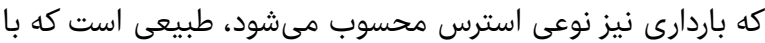

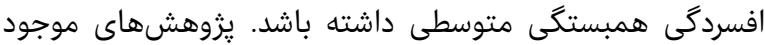

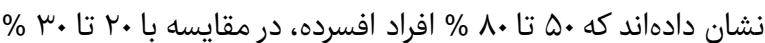

دردسترس انتخاب شدند. معيارهاى ورود شامل داشتن حداقل

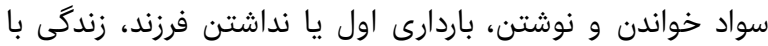

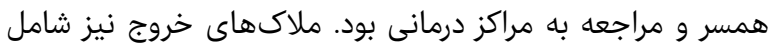

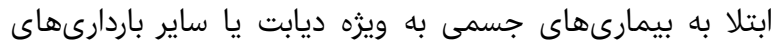

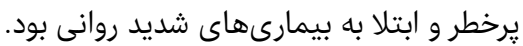

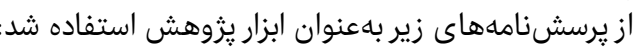

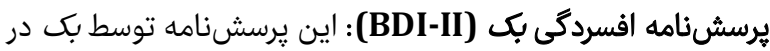

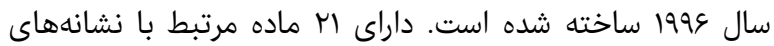

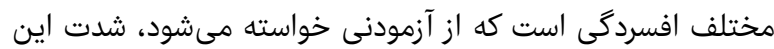

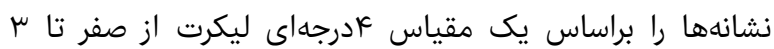

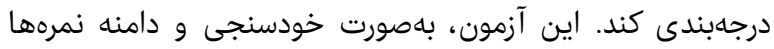

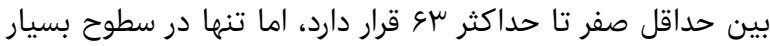

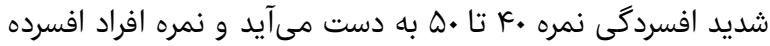

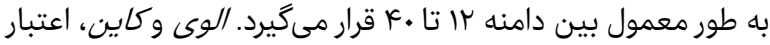

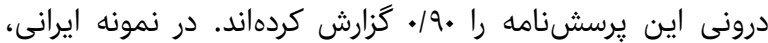

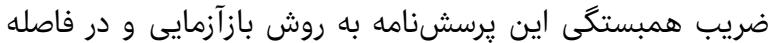

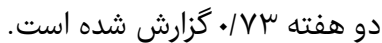

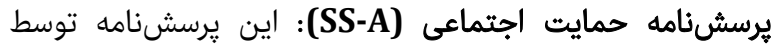

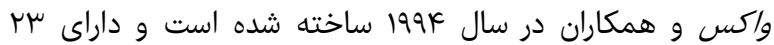

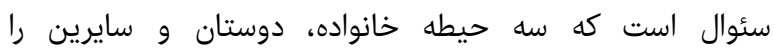

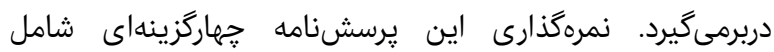

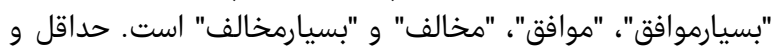

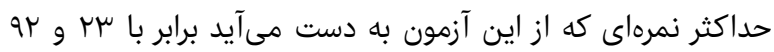

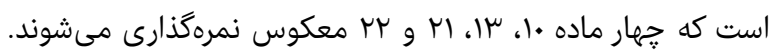

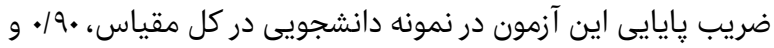

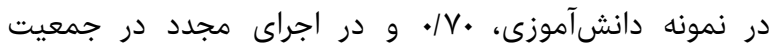

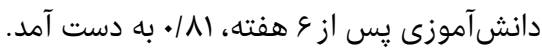

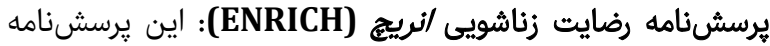

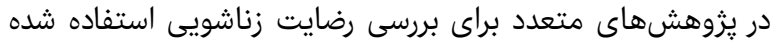

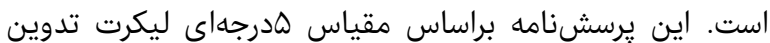

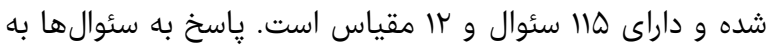

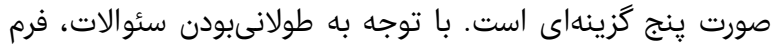

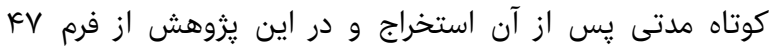

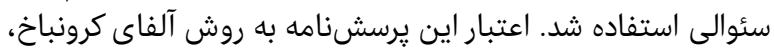

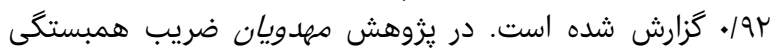

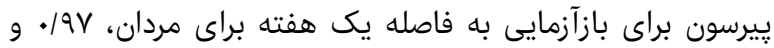

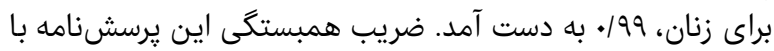

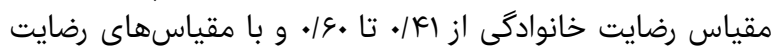

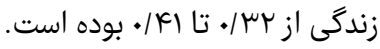

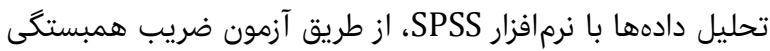

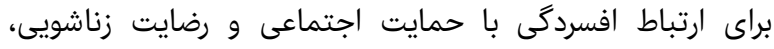

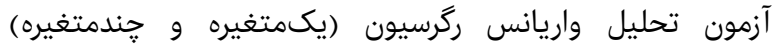

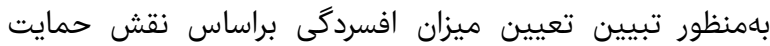
اجتماعى و رضايت زناشويى انجام شد. تبنين

\section{بافتهها}

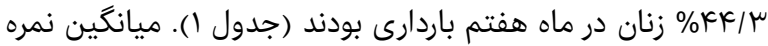
افسردگى زنان، Ir/VK

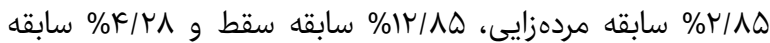

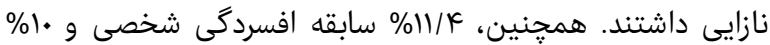
سابقه خانوادگى افسردگى داشتنى داشند 
همبستگى معكوس معنىدارى دارد و حمايت اجتماعى نسبت به

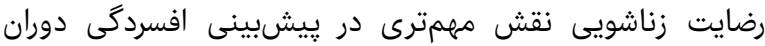

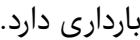

تشكر و قدردانى: موردى از سوى نويسندكان ذكر نشده است.

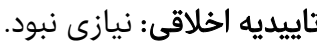

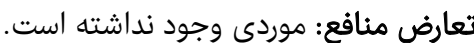

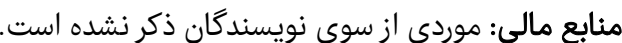

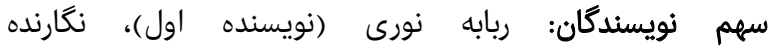

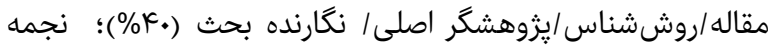

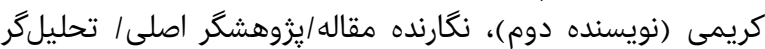

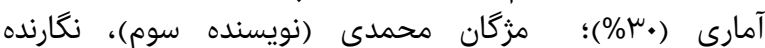

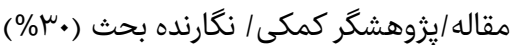

منابع

1- Ali NS, Azam IS, Ali BS, Tabbusum G, Moin SS Frequancy and associated factors for anxiety and depression in pregnant women: A hospital-based crosssectional study. Sci World J. 2012;2012:653098.

2- Evans J, Heron J, Francomb H, Oke S, Golding J. Cohort study of depressed mood during pregnancy and after chilfbirth. BMJ. 2001;323(7307):257-60.

3- Milgrom J, Gemmill AW, Bilszta JL, Hayes B, Barnett B, Brooks J, et al. Antenatal risk factors for postnatal depression: A large prospective study. J Affect Disord. 2008;108(1-2):147-57.

4- Rich-Edwards JW, Kleinman K, Abrams A, Harlow BL, McLaughlin TJ, Joffe $\mathrm{H}$ et al. Sociodemographic predictors of antenatal and postpartum depressive symptoms among women in medical group practice. J Epidemiol Community Health. 2006;60(3):221-7.

5- Heron J, O'Conner TG, Evans J, Goling J, Glover V, ALSPAC Study Team. The course of anxiety and depression through pregnancy and the postpartum in a community sample. J Affect Disord. 2004;80(1):65-73.

6- Thiagayson P, Krishnaswamy G, Lim ML, Sung SC, Haley CL, Fung DS, et al. Depression and anxiety in Singaporean high-risk pregnancies: Prevalence and screening. Gen Hosp Psychiatry. 2013;35(2):112-6.

7- Farzad M, GhaziMirsaid SB. Study of type of delivery with incidence of early depressive symptoms after delivery. shahidbeheshti J Med Sci. 2005;29(4):331-5 [Persian]

8- Verreault N, DaCosta D, Marchand A, Ireland K, Dritas $\mathrm{M}$, Khalife S. Rates and risk factors associated with depressive symptoms during pregnancy and with postpartum onset. J Psychosom Obstet Gynaecol. 2014;35(3):84-91.

9- Bennett HA, Einarson A, Taddio A, Koren G, Einarson TR. Prevalence of depression during pregnancy: Systemic review. Obstet Gynecol. 2004:103(4):698-709. 10- Gavin NI, Gaynes BN, Lohr KN, Meltzer-broy S, Gartlenhner G, Swinson T. Perinatal depression: A systematic review of prevalence and incidence. Obstet Gynecol. 2005;106(5 Pt 1):1071-83.

11- Oates M. Sucide: The leading cause of maternal death. Br J Psychiatry. 2003;183(4):279-81.

12- O'Connor TG, Heron J, Glover V; Alspac Study Team. Antenatal anxiety predicts child behavioral/emotional problems independently of postnatal depression. J Am Acad Child Adolesc Psychiatry. 2002:41(12):1470-7. 13- Straub H, Cross J, Curtis S, Iverson S, Jacobsmeyer M,
اف إبابه نورى و همكاران

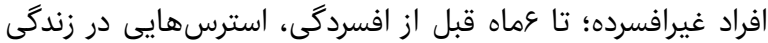

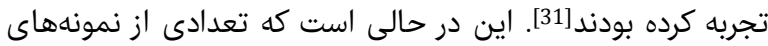

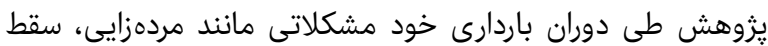

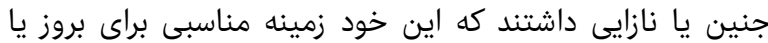

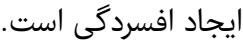

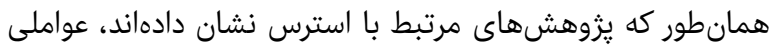

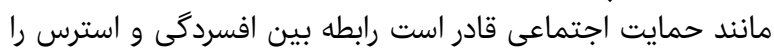

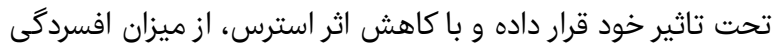

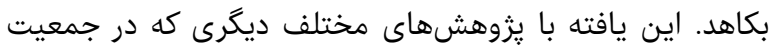

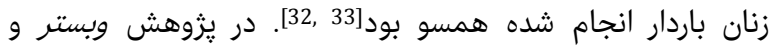

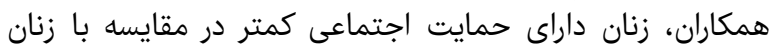

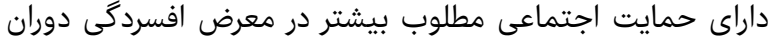

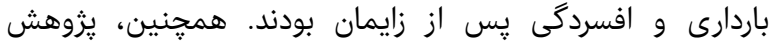

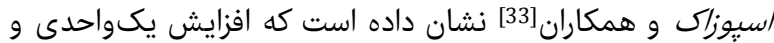

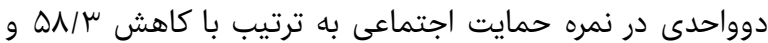

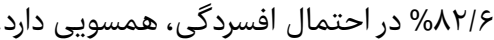

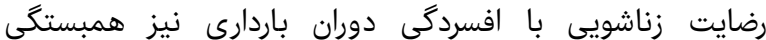

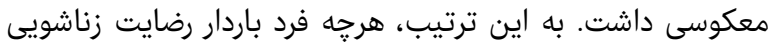

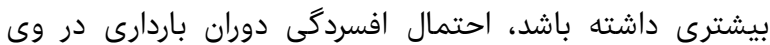

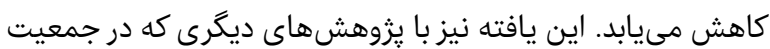

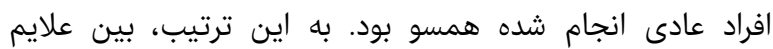

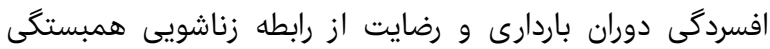

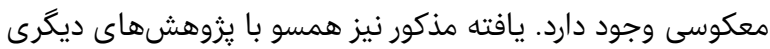

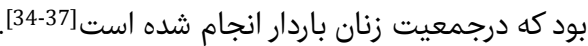

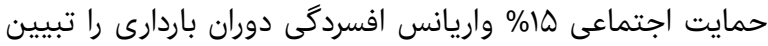

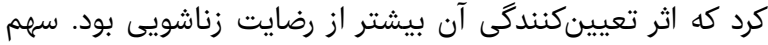

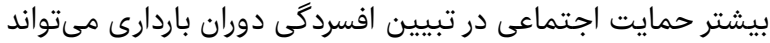

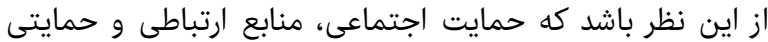

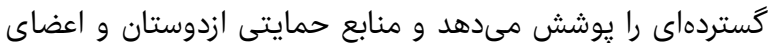

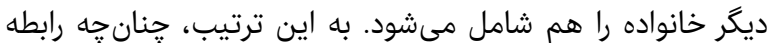

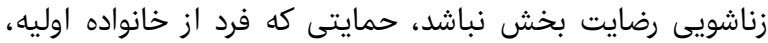

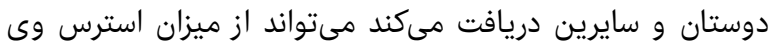

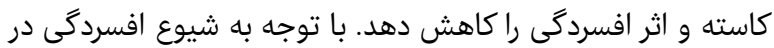

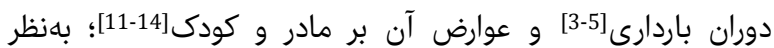

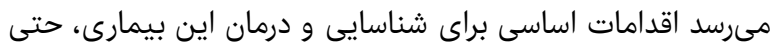

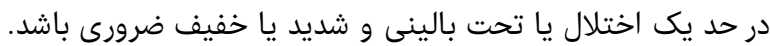

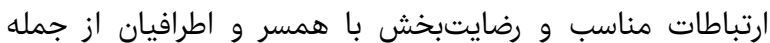

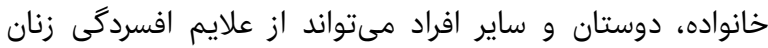

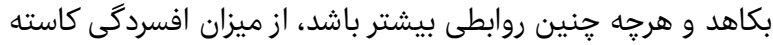

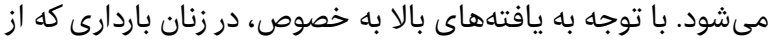

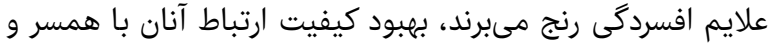

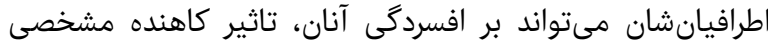

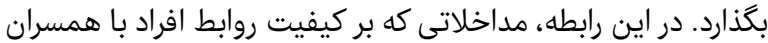

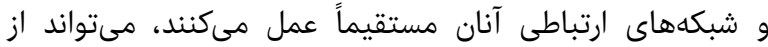

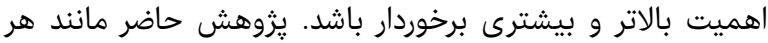

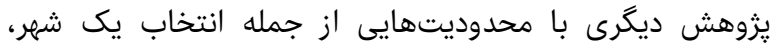

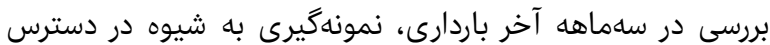

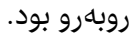

نتيجهگيرى دوران باردارى با حمايت اجتماعى و رضايت زناشويى، 
رابطه افسردگى دوران باردارى با حمايت اجتماعى و رضايت زناشويى

Psychological Association; 2001. pp. 3-24.

26- Siu BWM, Leung SSL, Ip P, Hung SF, O'Hara MW. Antenatal risk factors for postnatal depression: A prospective study of Chinese women at maternal and child health centres. BMC Psychiatry. 2012;12:22.

27- Schatz DB, Hsiao MC, Liu CY. Antenatal depression in East Asia: A review of the literature. Psychiatry Investig. 2012;9(2):111-8.

28- Senturk V, Abas M, Berksun 0, Stewart R. Social support and antenatal depression in extended and nuclear family environments in Turkey: A cross-sectional survey. BMC Psychiatry. 2011;11:48.

29- HadizadeTalasaz F, Bahri N, Tavakollizadeh J. Comparison of postpartum depression after natural delivery and emergency sezarian in first pregnant women who refer to "22 Bahman Hospital" of Gonabad city (2003). J Kermanshah Univ Med Sci. 2004;8(4):2130. [Persian]

30- Aghapour M, Mahmoudi A. comparison of postpartum depression between occupatied women and householders and it's relationship to social support and marital adjustment. J Women Stud fam. 2008; 1(4):9-32[Persian]

31- Kendler KS, Karkowski LM, Prescott CA. Causal relationship between stressful life events and the onset of major depression. Am J Psychiatry. 1999;156(6):83741.

32- Webster J, Linnane JW, Dibley LM, Hinson JK, Starrenburg SE, Roberts JA. Measuring social support in pregnancy: Can it be simple and meaningful?. Bitrh. 2000;27(2):97-101.

33- Spoozak L, Gotman N, Simth MV, Belanger K, Yonkers KA. Evaluation of a social support measure that may indicate risk of depression during pregnancy $\mathrm{J}$ Affect Disord. 2009;114(1-3):216-23.

34- Bakhshi H, Asadpor M, khodadadizade A. Relationships between marital satisfaction with depression in couples. Sci J Qazvin Univ Med Sci. 2007;11(2):37-43. [Persian]

35- Sayadi A. Study of scientific model of personality traits (Big Five), coping styles and mental health with marital satisfaction [Dissertation]. Tehran: Kharazmi University; 2007. [Persian]

36- Omidvar S, Khairkhah F, Azimi Urimi H. Depression in pregnancy and related factors. Med J Hormozgan Univ .2007;11(4):213-219. [Persian]

37- Buruns DD, Sayers SL, Moras K. Intimate realationships and depression: Is there a causal connection?. J Consult Clin Psychol. 1994;62(5):1033-43.
Anderson C, et al. Proactive nursing: The evolution of a task force to help women with postpartum depression. MCN Am J Matern Child Nurs. 1998:23(5):262-5.

14. Adler J, Fink N, Bitzer J, Hosli I, Holzgreve W. Depression and anxiety during pregnancy: A risk factor for obstetric, fetal and neonatal outcome? A critical review of the literature. J Matern Fetal Neonatal Med. 2007;20(3):189-209.

15- Robertson E, Grace S, Wallington T, Stewart DE. Antenatal risk factors for postpartum depression: A synthesis of recent literature. Gen Hosp Psychiatry. 2004;26(4):289-95.

16- Glover V, O'Connor TG, Heron J, Golding J; ALSPAC Study Team. Antenatal maternal anxiety is linked with atypical handedness in the child. Early Hum Dev. 2004;79(2):107-18.

17- Mohamad KI, Gamble J, Greedy DK. Prevalence and factors associated with development of antenatal and postnatal depression among Jordanian women. Midwifery. 2011;27(6):e238-45.

18- Faisal-Cury A, Menezes PR. Antenatal depression strongly predicts postnatal depression in primary health care. Rev Bras Psiquiatr. 2012;34(4):446-50.

19- Leigh B, Milgrom J. Risk factors for antenatal depression, postnatal depression and parenting stress. BMC Psychiatry. 2008;8:24.

20- Chittleborough CR, Lawlor DA, Lynch JW. Prenatal prediction of poor maternal and offspring outcomes: Implications for selection into intensive parent support programs. Matern Child Health J. 2012;16(4):909-20.

21- Jeong HG, Lim JS, Lee MS, Kim SH, Jung IK, Joe SH. The association of psychosocial factors and obstetric history with depression in pregnant women: Focus on the role of emotional support. Gen Hosp Psychiatry. 2013;35(4):354-8.

22- Zhang S, Ding Z, Liu H, Chen Z, Wu J, Zhang Y, Yu Y. Association between mental stress and gestational hypertension/preeclampsia: A meta-analysis. Obstet Gynecol Surv. 2013;68(12):825-34.

23- Sarafino EP, Smith EP. Health psychology: Biopsychosocial interaction. New York: Wiley. 2014. $560 \mathrm{p}$.

24- Rosand GMB, Slinning K, Eberhard-Gran M, Roysamb E, Tambs K. The buffering effect of relationship satisfaction on emotional distress in couples. BMC Public Health. 2012;12:66.

25- Whisman MA. The association between depression and marital dissatisfaction. In: Marital and Famiy processes in depression. Washington DC: American 